\title{
Recent advances in fragment-based computational drug design: tackling simultaneous targets/biological effects
}

\author{
Alejandro Speck-Planche*,1 \\ ${ }^{1}$ Research Program on Biomedical Informatics (GRIB), Hospital del Mar Medical Research Institute (IMIM), Barcelona 08003, Spain \\ *Author for correspondence: Tel.: + 34678042 076; alejspivanovich@gmail.com \\ "Multiscale de novo drug design is a new paradigm that combines mtk-QSBER models, chemical
reasoning and a computational variant of FBDD named fragment-based topological design."
}

First draft submitted: 4 June 2018; Accepted for publication: 4 June 2018; Published online:

30 July 2018

Keywords: ADME • fragment-based drug discovery • integrative • mtk-QSBER models • multiscale de novo drug design

The pharmaceutical industry has provided a plethora of drugs to cure a multitude of diseases. However, with a period of time set to be 12-17 years, and a cost around US $\$ 3$ billion per new drug approval [1], drug discovery still remains a very complex, time-consuming and expensive process. In fact, it is now clearer that developing a new drug within the pharmaceutical sector may have become financially unsustainable [2].

Fragment-based drug design (FBDD) is a promising approach that has demonstrated a great potential for improving lead optimization, contributing to diminishing the high attrition rates at all stages in drug development; it is an approach that identifies small molecular fragments with weak affinity for a biomolecular target of interest and assembles them into full drug-like compounds. At the same time, FBDD has benefited from the application of chemoinformatics and computational tools [3].

However, as any modern drug discovery approach, and despite the success in providing effective therapeutic agents, FBDD has been considerably affected by several factors. First, the chemical space to be covered in the search for small-molecule drugs is huge [3], being in the order of $10^{60}$ organic molecules. Second, as huge amounts of data exponentially accumulate in the domains of the biological, chemical and clinical sciences, it is clear that drug discovery should be analyzed as a multiscale optimization problem, thus being focused on data integration beyond the molecular level [4]. Third, many biological entities that cause diseases (bacteria, cancer cell lines, and others), have become resistant to the drugs originally developed to eradicate them [5-7]. Fourth, many diseases are difficult to treat due to their multi-factorial nature, which involves genetic, environmental or complex factors, the latter resulting from interactions between (multiple) genetic and environmental factors [8]. Last, regardless of the pharmacological activity and the drug design approach used, drugs are prone to have serious side effects [9].

The failure of the pharmaceutical industry in designing truly safe drugs can be summarized in one phrase: due to the reliance on the filtering-based approach for developing drugs, it is not possible to simultaneously assess the desired dosage required at the different stages of the drug discovery process (from early development to preclinical studies and clinical trials). Consequently, any drug candidate with very low-inhibitory potency against an extensive panel of off-target proteins almost never translates into a drug with the safety profiles according to those previous assays.

In a more ideal context, a drug must have a potent biological activity, desirable absorption, distribution, metabolism and elimination (ADME) properties, and very low toxicity; all these biological effects must exist at both in vitro and in vivo levels, and regardless of the biological targets; here, the term 'biological target' refers to all the scales of biologically relevant entities against which a drug/chemical is tested, i.e., proteins, microorganisms, cells, tissues, organs, organ systems and organisms. 
The prime goal is to design a drug with high efficacy, excellent ADME profiles and with no (or a very few slight) side effects. The scientific community is aware of three main aspects that are already shaping the drug development environment: the potential of FBDD, the essential role of chemoinformatics in drug discovery campaigns and the increasing need to use multiscale integrative approaches. This work briefly discusses the role of the multiscale chemoinformatic models in drug discovery, and how they have tackled the problem of simultaneously predicting multiple biological effects against many different biological targets. Here, the joint application of multiscale chemoinformatic models and concepts derived from FBDD is analyzed as a new term: multiscale de novo drug design.

\section{Multiscale chemoinformatic models: great allies of drug discovery}

When referring to the term 'multiscale chemoinformatic models', it is necessary to emphasize that this term is used only for those models that can truly integrate data, for example, models capable of simultaneously predicting biological activity, toxic effects and ADME parameters at in vitro and in vivo levels against multiple biological targets. To date, there are only two kinds of models that can perform this task: multitasking models for quantitative structure-biological effect relationships (mtk-QSBER) [10], and perturbation theory-based models for quantitative structure-property relationships [11]. However, for the reason of space, and simplicity of application of mtk-QSBER models over their quantitative structure-property relationships counterparts, only the first ones will be analyzed here.

The mtk-QSBER models are multiscale chemoinformatic models which apply transformations to the molecular descriptors initially calculated for each molecule in a dataset. Such transformations are made by using the BoxJenkins operators reported in time-series analysis. In this sense, a Box-Jenkins operator used in time-series calculate the successive average values of a characteristic of a system at different intervals of time or seasons [11]. In multiscale modeling, the Box-Jenkins approach does not include the time domain, and therefore, it calculates the average of any molecular descriptor for all drugs/chemicals with a specific response in an assay under a subset of experimental conditions. After, the aforementioned average is subtracted from the original descriptor of each drug chemical, yielding a deviation descriptor; such descriptor measures how much a drug/chemical structurally deviates from a set of compounds tested under the same experimental conditions. For this reason, developing an mtk-QSBER model implies that any molecule may appear in the dataset (and therefore can be predicted) as many times as different experimental conditions have been used to test it; the value of the deviation descriptor for the same molecule will likely be different for each different assay condition. Thus, applications of the mtk-QSBER methodology have been evidenced in the last 5 years in different fields of research, which include (but are not limited to) neurosciences [12], immunotoxicology [13] and antibacterial research [14].

\section{Multiscale de novo drug design: where fragment-based drug design \& multitasking models for quantitative structure-biological effect relationships converge}

It is clear that the mtk-QSBER models have been capable of tackling problems in drug discovery such as the simultaneous prediction of multiple effects against many different biological targets. However, these multiscale chemoinformatic models have limitations, which are common to most of the computational models used to perform prospective predictions. From one side, mtk-QSBER models are descriptor-dependent, and therefore, their performance will be restricted to the nature of the molecular descriptors used to develop them. On the other hand, there are no universal descriptors, in other words, the molecular descriptors created to date are not able to fully characterize the chemical structures of the molecules. Consequently, even when the reliability of the predictions of the mtk-QSBER models is assessed (e.g., applicability domain methods, consensus/voting approaches), in the end, mtk-QSBER models will reliably predict to some extent. Additionally, due to the large numbers of effects and biological targets from which the mtk-QSBER models are constructed, performing virtual screening in massive databases can be computationally challenging. Thus, it is imperative to optimize the chemical space that needs to be screened. In this sense, we can benefit from the integrative power of the mtk-QSBER models and the capabilities of FBDD to perform optimization to the chemical structure. A new term then emerges: multiscale de novo drug design.

Multiscale de novo drug design is a new paradigm that combines mtk-QSBER models, chemical reasoning [15] and a computational variant of FBDD named fragment-based topological design [16]. Here, it should be emphasized that through chemical reasoning, multiscale de novo drug design rescues the idea regarding the importance of interpreting the molecular descriptors of a model from a physicochemical/structural point of view; this aspect 
is generally neglected in models focused on 'big data'. By assembling different suitable molecular fragments, the purpose of the multiscale de novo drug design methodology is to create a series of new molecules that exhibit high biological activity, low toxicity, and desirable ADME properties at both in vitro and in vivo levels. These designed molecules have acceptable synthetic accessibility and fall within the applicability domain of the mtk-QSBER models. Currently, multiscale de novo drug design has been successfully applied to the discovery of anti-hepatitis C agents [17], the design of potential antibiotics against Gram-negative bacteria [18] and the generation of anti-HIV compounds [19].

\section{Conclusion}

Targeting the chemical space to find optimal drugs is a goal of paramount importance in drug discovery. By merging the mtk-QSBER philosophy with ideas and concepts borrowed from FBDD, multiscale de novo drug design offers a promising solution to solving this challenging task. Through multiscale de novo drug design, it is possible to handle the huge amounts of chemical, biological and clinical data, and subsequently, extract the chemical features responsible for the improvement of the properties that any drug-like molecule must have. Although in its beginnings, multiscale de novo drug design has emerged as an encouraging approach that opens new horizons toward the discovery of novel, more effective and safer therapeutic agents.

\section{Financial \& competing interests disclosure}

A Speck-Planche acknowledges the Spanish Juan de la Cierva program (Grant: FJCI-2015-25572) for the financial support. The author has no other relevant affiliations or financial involvement with any organization or entity with a financial interest in or financial conflict with the subject matter or materials discussed in the manuscript apart from those disclosed.

No writing assistance was utilized in the production of this manuscript.

\section{References}

1. DiMasi JA, Grabowski HG, Hansen RW. Innovation in the pharmaceutical industry: new estimates of R\&D costs. J. Health Econ. 47, 20-33 (2016).

2. Liotta D, Painter G. Academic drug development: the DRIVE model. ACS Med. Chem. Lett. 9(5), 403-407 (2018).

3. Jahnke W, Erlanson DA. Fragment-based approaches in drug discovery. Wiley-VCH Verlag GmbH \& Co. KGaA, Weinheim, Germany (2006).

4. Sanz F, Pognan F, Steger-Hartmann T et al. Legacy data sharing to improve drug safety assessment: the eTOX project. Nat. Rev. Drug Discov. 16(12), 811-812 (2017).

5. Friedman R. Drug resistance in cancer: molecular evolution and compensatory proliferation. Oncotarget 7(11), 11746-11755 (2016).

6. Vasoo S, Barreto JN, Tosh PK. Emerging issues in Gram-negative bacterial resistance: an update for the practicing clinician. Mayo Clin. Proc. 90(3), 395-403 (2015).

7. Holohan C, Van Schaeybroeck S, Longley DB, Johnston PG. Cancer drug resistance: an evolving paradigm. Nat. Rev. Cancer 13(10), $714-726$ (2013).

8. Stolk RP, Rosmalen JG, Postma DS et al. Universal risk factors for multifactorial diseases: LifeLines, a three-generation population-based study. Eur. J. Epidemiol. 23(1), 67-74 (2008).

9. Kuhn M, Letunic I, Jensen LJ, Bork P. The SIDER database of drugs and side effects. Nucleic Acids Res. 44(D1), D1075-D1079 (2016).

10. Speck-Planche A, Cordeiro MN. Multitasking models for quantitative structure-biological effect relationships: current status and future perspectives to speed up drug discovery. Expert Opin. Drug Discov. 10(3), 245-256 (2015).

11. Gonzalez-Diaz H, Herrera-Ibata DM, Duardo-Sanchez A, Munteanu CR, Orbegozo-Medina RA, Pazos A. ANN multiscale model of anti-HIV drugs activity vs AIDS prevalence in the US at county-level based on information indices of molecular graphs and social networks. J. Chem. Inf. Model. 54(3), 744-755 (2014).

12. Romero-Duran FJ, Alonso N, Yanez M, Caamano O, Garcia-Mera X, Gonzalez-Diaz H. Brain-inspired cheminformatics of drug-target brain interactome, synthesis and assay of TVP1022 derivatives. Neuropharmacology 103, 270-278 (2016).

13. Tenorio-Borroto E, Penuelas-Rivas CG, Vasquez-Chagoyan JC et al. Model for high-throughput screening of drug immunotoxicity: study of the antimicrobial G1 over peritoneal macrophages using flow cytometry. Eur. J. Med. Chem. 72, 206-220 (2014).

14. Speck-Planche A, Cordeiro MN. Simultaneous virtual prediction of anti-Escherichia coli activities and ADMET profiles: a chemoinformatic complementary approach for high-throughput screening. ACS Comb. Sci. 16(2), 78-84 (2014).

15. Speck-Planche A, Cordeiro MN. Chemoinformatics for medicinal chemistry: in silico model to enable the discovery of potent and safer anti-cocci agents. Future Med. Chem. 6(18), 2013-2028 (2014). 
16. Speck-Planche A, Cordeiro MN. Fragment-based in silico modeling of multitarget inhibitors against breast cancer-related proteins. Mol. Divers. 21(3), 511-523 (2017).

17. Speck-Planche A, Cordeiro MN. Speeding up early drug discovery in antiviral research: a fragment-based in-silico approach for the design of virtual anti-hepatitis C leads. ACS Comb. Sci. 19(8), 501-512 (2017).

18. Speck-Planche A, Cordeiro MN. De novo computational design of compounds virtually displaying potent antibacterial activity and desirable in vitro ADMET profiles. Med. Chem. Res. 26(10), 2345-2356 (2017).

19. Kleandrova VV, Speck Planche A. Multitasking model for computer-aided design and virtual screening of compounds with high anti-HIV activity and desirable ADMET properties. In: Multiscale Approaches in Drug Discovery: From Empirical Knowledge to In Silico Experiments and Back. Speck-Planche A (Ed.) Elsevier, Oxford, UK, 55-81 (2017). 